PROCEEDINGS OF THE

AMERICAN MATHEMATICAL SOCIETY

Volume 130, Number 10, Pages 2971-2983

S 0002-9939(02)06445-6

Article electronically published on March 12, 2002

\title{
SELF-COMMUTATOR INEQUALITIES IN HIGHER DIMENSION
}

\author{
MIRCEA MARTIN
}

(Communicated by Joseph A. Ball)

\begin{abstract}
Three natural multi-dimensional substitutes for the self-commutator of a Hilbert space operator are introduced and generalizations of Putnam's inequality to tuples of operators with semidefinite self-commutators are indicated. In addition, a Riesz transform model is developed and investigated.
\end{abstract}

\section{INTRODUCTION}

This article is concerned with two Putnam type inequalities in higher dimension. In its one-dimensional form, Putnam's inequality points out that the area of the spectrum of a seminormal Hilbert space operator dominates the norm of the selfcommutator of that operator. The original proof dates back to the early 1970s and is given in [30]. Simplified forms and several new proofs have been discovered in the meantime by different authors. For an insight and a better historical perspective we refer to the monographs [29, [6, 8], [36, and [18. We would like to mention that the proofs discussed in [6] and [36] rely on the singular integral models developed by Xia [35], Pincus [26, Kato [14, and Muhly [24] (see also [27] and [28]). A simple proof for subnormal operators has been found by Axler and Shapiro [3]. Their work relates Putnam's result to an inequality due to Alexander [1] and reveals connections between seminormality, one-variable complex analysis, and harmonic analysis. The generalization established in [17] of Alexander's inequality in the Clifford analysis setting suggests the use of some tools pertaining to this field in generalizing Putnam's inequality.

This task clearly requires a good concept of joint seminormality in higher dimension. Steps towards the development of a multi-variable theory are indicated in [2, 7], 9], 10], 11, 12, 16, 19], 21], and [37, to mention just a few of the contributors in this area. Parts of the present article emphasize the viewpoint advanced in [16] and [19], where joint seminormality is investigated based on a Bochner-Weitzenböck and two Bochner-Kodaira identities associated to systems of operators. This approach brings in several techniques customarily employed in spin geometry and Clifford analysis. We should notice that Clifford analysis is just the kind of function theory that matches spin geometry. Excellent presentations of these topics can be found in [4], [5], [13], 15], and [23]. Specific applications of Clifford analysis to operator theory related in part to our article are presented in 22] and [32] and in several articles included in the volume 31] edited by J. Ryan.

Received by the editors June 27, 2000 and, in revised form, May 7, 2001.

1991 Mathematics Subject Classification. Primary 47B20, 42 B20.

Key words and phrases. Hyponormal operators, Putnam's inequality, Riesz transforms.

(C)2002 American Mathematical Society 
The article has two sections that deal with self-commutators in higher dimension, the Riesz transform model and Putnam type inequalities.

\section{SELF-COMmUtATORS IN Higher Dimension}

This section singles out multi-dimensional substitutes for the self-commutator of a Hilbert space operator. We address several related issues such as the existing definitions of joint seminormality, Bochner-Kodaira identities in multi-variable operator theory, and the Riesz transform model.

2.1. We let $\mathcal{H}$ be a complex infinite-dimensional Hilbert space and denote by $\mathcal{L}(\mathcal{H})$ the $C^{*}$-algebra of all bounded linear operators on $\mathcal{H}$. The adjoint of $T \in \mathcal{L}(\mathcal{H})$ is denoted by $T^{*}$ and the real and imaginary parts of $T$ are defined as $\operatorname{Re}(T)=$ $\left(T+T^{*}\right) / 2, \operatorname{Im}(T)=\left(T-T^{*}\right) / 2 \sqrt{-1}$.

If $T=\left(T_{1}, T_{2}, \ldots, T_{n}\right)$ is an $n$-tuple of operators in $\mathcal{L}(\mathcal{H})$, we introduce the left and right self-commutator of $T$ as the $n \times n$ operator matrices given by

$$
\mathcal{C}_{\mathrm{L}}(T)=\left(\left[T_{i}, T_{j}^{*}\right]\right)_{i, j=1}^{n}, \quad \mathcal{C}_{\mathrm{R}}(T)=\left(\left[T_{i}^{*}, T_{j}\right]\right)_{i, j=1}^{n},
$$

and regarded as operators on the space $\mathbb{C}^{n} \otimes \mathcal{H}$.

We next take the self-adjoint $n$-tuples $X=\left(X_{1}, X_{2}, \ldots, X_{n}\right)$ and $Y=\left(Y_{1}, Y_{2}, \ldots\right.$, $\left.Y_{n}\right)$, where $X_{i}=\operatorname{Re}\left(T_{i}\right)$ and $Y_{i}=\operatorname{Im}\left(T_{i}\right), 1 \leq i \leq n$, and introduce

$$
\mathcal{C}(X, Y)=\sqrt{-1}\left(\left[X_{i}, Y_{j}\right]+\left[X_{j}, Y_{i}\right]\right)_{i, j=1}^{n} .
$$

In the case $n=1$ we obviously have $\mathcal{C}_{\mathrm{R}}(T)=\mathcal{C}(X, Y)=-\mathcal{C}_{\mathrm{L}}(T)$, so basically we are dealing with a single commutator. If $n \geq 2$, these properties are no longer true, and although the three so-defined commutators are related to each other, they produce different operators on $\mathbb{C}^{n} \otimes \mathcal{H}$. Specifically, $\mathcal{C}_{\mathrm{L}}(T)$ and $\mathcal{C}_{\mathrm{R}}(T)$ are in general linearly independent, $\mathcal{C}_{\mathrm{R}}(T)$ is the formal transpose of $-\mathcal{C}_{\mathrm{L}}(T)$, and

$$
\mathcal{C}(X, Y)=\frac{1}{2}\left(\mathcal{C}_{\mathrm{R}}(T)-\mathcal{C}_{\mathrm{L}}(T)\right)
$$

The terminology used below is the same as in [16] and [19].

2.2. Definition. An $n$-tuple $T$ of operators on $\mathcal{H}$ is called left or right seminormal provided $\mathcal{C}_{\mathrm{L}}(T)$ or $\mathcal{C}_{\mathrm{R}}(T)$ is semidefinite as an operator on $\mathbb{C}^{n} \otimes \mathcal{H}$. In the case $\mathcal{C}_{\mathrm{L}}(T) \geq 0$ or $\mathcal{C}_{\mathrm{L}}(T) \leq 0$, the $n$-tuple $T$ is said to be left cohyponormal or left hyponormal, respectively, and when $\mathcal{C}_{\mathrm{R}}(T) \geq 0$ or $\mathcal{C}_{\mathrm{R}}(T) \leq 0$, the $n$-tuple $T$ is called right hyponormal or right cohyponormal, respectively.

If $n=1$, we recover the standard definitions of seminormal, hyponormal, and cohyponormal operators. The concepts of joint hyponormality, joint cohyponormality, and $t$-hyponormality studied in [2] and 37] correspond in our terminology to left hyponormality, left cohyponormality, and right hyponormality, respectively.

2.3. Definition. A pair of two self-adjoint $n$-tuples $X$ and $Y$ of operators on $\mathcal{H}$ is called jointly seminormal provided its commutator $\mathcal{C}(X, Y)$ is semidefinite as an operator on $\mathbb{C}^{n} \otimes \mathcal{H}$. In the case when $\mathcal{C}(X, Y) \geq 0$ or $\mathcal{C}(X, Y) \leq 0$, the pair $(X, Y)$ is said to be jointly hyponormal or jointly cohyponormal, respectively.

Since $\mathcal{C}_{\mathrm{L}}(T)=\mathcal{C}_{\mathrm{R}}\left(T^{*}\right)$, where $T^{*}=\left(T_{1}^{*}, T_{2}^{*}, \ldots, T_{n}^{*}\right)$, the adjoint of a left cohyponormal, or hyponormal, tuple is right hyponormal, or cohyponormal. We note that the self-adjoint pair $(X, Y)$ associated with an $n$-tuple $T$ which is simultaneously left and right hyponormal, or left and right cohyponormal, turns out to be jointly hyponormal, or jointly cohyponormal, as it follows from (2.3). 
2.4. Definitions 2.2 and 2.3 gain a better motivation in conjunction with two Bochner-Kodaira type identities for $n$-tuples of operators on $\mathcal{H}$. To establish them, we employ the complex Clifford algebra $\mathfrak{A}_{2 n}=\mathfrak{A}_{2 n}(\mathbb{C})$ over the Euclidean space $\mathbb{R}^{2 n} \cong \mathbb{C}^{n}$. Specifically, $\mathfrak{A}_{2 n}$ is the $C^{*}$-algebra generated by the standard orthonormal basis $\left\{e_{1}, e_{2}, \ldots, e_{2 n-1}, e_{2 n}\right\}$ for $\mathbb{R}^{2 n}$, subject to the relations $e_{k} e_{l}+e_{l} e_{k}=-2 \delta_{k l}, 1 \leq k, l \leq 2 n$, with the involution defined by $e_{k}^{*}=-e_{k}$, $1 \leq k \leq 2 n$. A more convenient set of generators can be introduced by setting $\varepsilon_{i}=\left(e_{i}-\sqrt{-1} e_{n+i}\right) / 2,1 \leq i \leq n$. We easily get $e_{i}=\varepsilon_{i}-\varepsilon_{i}^{*}, e_{n+i}=\sqrt{-1}\left(\varepsilon_{i}+\varepsilon_{i}^{*}\right)$, $1 \leq i \leq n$, and the following two important properties:

$$
\varepsilon_{i} \varepsilon_{j}+\varepsilon_{j} \varepsilon_{i}=0, \quad \varepsilon_{i} \varepsilon_{j}^{*}+\varepsilon_{j}^{*} \varepsilon_{i}=\delta_{i j}, \quad 1 \leq i, j \leq n .
$$

We suppose next that $T=\left(T_{1}, T_{2}, \ldots, T_{n}\right)$ is an $n$-tuple of operators on $\mathcal{H}$ and associate to it the operator $\mathcal{D}(T)$ in $\mathfrak{A}_{2 n} \otimes \mathcal{L}(\mathcal{H})$ given by

$$
\mathcal{D}(T)=\sum_{i=1}^{n} \varepsilon_{i} \otimes T_{i}
$$

Using $\mathcal{D}(T)$ and its adjoint $\mathcal{D}^{*}(T)$ we form the complex laplacian of $T$,

$$
\Delta(T)=\mathcal{D}(T) \mathcal{D}^{*}(T)+\mathcal{D}^{*}(T) \mathcal{D}(T) .
$$

In addition, we introduce the left and right connection laplacians of $T$ defined by

$$
\Delta_{\mathrm{L}}^{\mathrm{c}}(T)=\sum_{i=1}^{n} T_{i}^{*} T_{i}, \quad \Delta_{\mathrm{R}}^{\mathrm{c}}(T)=\sum_{i=1}^{n} T_{i} T_{i}^{*} .
$$

We are now in a position to set up two Bochner-Kodaira type identities that relate the three just defined laplacians. Direct calculations based on (2.4) show that

$$
\Delta(T)=I \otimes \Delta_{\mathrm{L}}^{\mathrm{c}}(T)+\mathcal{R}_{\mathrm{L}}(T)
$$

and

$$
\Delta(T)=I \otimes \Delta_{\mathrm{R}}^{\mathrm{c}}(T)+\mathcal{R}_{\mathrm{R}}(T),
$$

where $I$ stands for the unit in $\mathfrak{A}_{2 n}$ and

$$
\mathcal{R}_{\mathrm{L}}(T)=\sum_{i, j=1}^{n} \varepsilon_{i} \varepsilon_{j}^{*} \otimes\left[T_{i}, T_{j}^{*}\right], \quad \mathcal{R}_{\mathrm{R}}(T)=\sum_{i, j=1}^{n} \varepsilon_{i}^{*} \varepsilon_{j} \otimes\left[T_{i}^{*}, T_{j}\right] .
$$

For more details and a geometric derivation of the identities $\left(\mathrm{BK}_{\mathrm{L}}\right)$ and $\left(\mathrm{BK}_{\mathrm{R}}\right)$ we refer the reader to [16. The point we want to make is that whenever $T$ is a commuting $n$-tuple, these identities are genuine Bochner-Kodaira identities set up on a hermitian Dirac bundle associated with $T$. This geometric approach naturally brings in the so-called Bochner's method that yields various vanishing theorems in spin geometry under appropriate positivity assumptions on the remainders in some specific Bochner-Weitzenböck or Bochner-Kodaira identities. The interested reader may find elaborate accounts on this subject in [4] and [15]. 
2.5. To implement Bochner's method in multi-variable operator theory, we need to examine the remainders $\mathcal{R}_{\mathrm{L}}$ and $\mathcal{R}_{\mathrm{R}}$ defined by (2.6). This could be done by using the space of complex spinors $\mathfrak{S}_{2 n}$ that provides the unique irreducible representation of the Clifford algebra $\mathfrak{A}_{2 n}$ (see [13] or [15]). As a Hilbert space, $\mathfrak{S}_{2 n}$ equals the complex exterior algebra $\Lambda^{*}\left(\mathbb{C}^{n}\right)=\bigoplus_{p=0}^{n} \Lambda^{p}\left(\mathbb{C}^{n}\right)$ over $\mathbb{C}^{n}$ equipped with the inner product inherited from $\mathbb{C}^{n}$. For each $\sigma \in \mathfrak{S}_{2 n}^{1}=\Lambda^{1}\left(\mathbb{C}^{n}\right)$ we let $\varepsilon(\sigma) \in \mathcal{L}\left(\mathfrak{S}_{2 n}\right)$ denote the operator of left exterior multiplication by $\sigma$. Its adjoint $\varepsilon(\sigma)^{*}$ is the operator of left interior multiplication by $\sigma$. For any $\sigma^{\prime}, \sigma^{\prime \prime} \in \mathfrak{S}_{2 n}^{1}$ we have

$$
\varepsilon\left(\sigma^{\prime}\right) \varepsilon\left(\sigma^{\prime \prime}\right)+\varepsilon\left(\sigma^{\prime \prime}\right) \varepsilon\left(\sigma^{\prime}\right)=0
$$

and

$$
\varepsilon\left(\sigma^{\prime}\right) \varepsilon\left(\sigma^{\prime \prime}\right)^{*}+\varepsilon\left(\sigma^{\prime \prime}\right)^{*} \varepsilon\left(\sigma^{\prime}\right)=\left\langle\sigma^{\prime}, \sigma^{\prime \prime}\right\rangle I,
$$

where $I$ stands for the identity operator on $\mathfrak{S}_{2 n}$. In particular, if $\left\{\sigma_{1}, \sigma_{2}, \ldots, \sigma_{n}\right\}$ denotes the standard orthonormal basis for $\mathbb{C}^{n}$ and $\mathbb{C}^{n}$ is identified with $\Lambda^{1}\left(\mathbb{C}^{n}\right)$, then we get that the operators $\varepsilon_{i}=\varepsilon\left(\sigma_{i}\right), 1 \leq i \leq n$, satisfy the basic properties (2.4). Consequently, $\mathfrak{S}_{2 n}$ becomes an $\mathfrak{A}_{2 n}$-module. As a matter of fact, the complex Clifford algebra $\mathfrak{A}_{2 n}$ is isomorphic to the full algebra $\mathcal{L}\left(\mathfrak{S}_{2 n}\right)$ and in what follows we are going to identify them.

We now return to the identities $\left(\mathrm{BK}_{\mathrm{L}}\right)$ and $\left(\mathrm{BK}_{\mathrm{R}}\right)$ and regard the two remainders $\mathcal{R}_{\mathrm{L}}$ and $\mathcal{R}_{\mathrm{R}}$ as operators on $\mathfrak{S}_{2 n} \otimes \mathcal{H}$. We convert $\mathfrak{S}_{2 n} \otimes \mathcal{H}$ into a graded space by setting $\mathfrak{S}_{2 n} \otimes \mathcal{H}=\bigoplus_{p=0}^{n} \mathfrak{S}_{2 n}^{p} \otimes \mathcal{H}$, where $\mathfrak{S}_{2 n}^{p}=\Lambda^{p}\left(\mathbb{C}^{n}\right), 0 \leq p \leq n$. With respect to this grading, both remainders $\mathcal{R}_{\mathrm{L}}$ and $\mathcal{R}_{\mathrm{R}}$ are homogeneous of degree zero. Their restrictions to the homogeneous subspaces $\mathfrak{S}_{2 n}^{p} \otimes \mathcal{H}$ are denoted by $\mathcal{R}_{\mathrm{L}}^{(p)}$ and $\mathcal{R}_{\mathrm{R}}^{(p)}, 0 \leq p \leq n$.

Further, we let $*: \mathfrak{S}_{2 n} \rightarrow \mathfrak{S}_{2 n}$ denote the Hodge star-operator that maps $\mathfrak{S}_{2 n}^{p}$ into $\mathfrak{S}_{2 n}^{n-p}$ for each $0 \leq p \leq n$, such that

$$
\sigma \wedge * \sigma=\|\sigma\|^{2} \sigma_{1} \wedge \sigma_{2} \wedge \cdots \wedge \sigma_{n}, \quad \sigma \in \mathfrak{S}_{2 n}^{p} .
$$

In particular, we have $* \sigma_{i}=(-1)^{i-1} \sigma_{1} \wedge \cdots \wedge \sigma_{i-1} \wedge \sigma_{i+1} \wedge \cdots \wedge \sigma_{n}, 1 \leq i \leq n$. By (2.6) we get the following explicit equations:

$$
\begin{aligned}
& \mathcal{R}_{\mathrm{L}}(T)\left(\sigma_{j} \otimes \xi\right)=\sum_{i=1}^{n} \sigma_{i} \otimes\left[T_{i}, T_{j}^{*}\right] \xi, \quad 1 \leq i \leq n, \xi \in \mathcal{H}, \\
& \mathcal{R}_{\mathrm{L}}(T)\left(* \sigma_{j} \otimes \xi\right)=\sum_{i=1}^{n} * \sigma_{i} \otimes\left[T_{i}^{*}, T_{j}\right] \xi, \quad 1 \leq i \leq n, \xi \in \mathcal{H}
\end{aligned}
$$

The already mentioned identification of $\mathfrak{S}_{2 n}^{1}$ with $\mathbb{C}^{n}$ and (2.7) show that the matrix representing $\mathcal{R}_{\mathrm{L}}^{(1)}(T)$ under this identification equals the left self-commutator $\mathcal{C}_{\mathrm{L}}(T)$ defined by (2.1). The isomorphism between $\mathfrak{S}_{2 n}^{n-1}$ and $\mathbb{C}^{n}$ that results by mapping $* \sigma_{i} \in \mathfrak{S}_{2 n}^{n-1}$ into $\sigma_{i} \in \mathbb{C}^{n}, 1 \leq i \leq n$, enables us to represent the operator $\mathcal{R}_{\mathrm{R}}^{(n-1)}(T)$ on $\mathfrak{S}_{2 n}^{n-1} \otimes \mathcal{H}$ as an operator matrix as well. As expected, by (2.8) that matrix turns out to be $\mathcal{C}_{\mathrm{R}}(T)$.

Therefore, we may now reformulate Definition 2.2. The next result points out that we can actually use $\mathcal{R}_{\mathrm{L}}(T)$ and $\mathcal{R}_{\mathrm{R}}(T)$ instead of $\mathcal{C}_{\mathrm{L}}(T)$ and $\mathcal{C}_{\mathrm{R}}(T)$ with no loss of generality. 
2.6. Proposition. Let $T=\left(T_{1}, T_{2}, \ldots, T_{n}\right)$ be an $n$-tuple of operators on $\mathcal{H}$.

(i) $\mathcal{C}_{\mathrm{L}}(T) \geq 0$ (resp. $\leq 0$ ) as an operator on $\mathbb{C}^{n} \otimes \mathcal{H}$ if and only if $\mathcal{R}_{\mathrm{L}}(T) \geq 0$ (resp. $\leq 0$ ) as an operator on $\mathfrak{S}_{2 n} \otimes \mathcal{H}$.

(ii) $\mathcal{C}_{\mathrm{R}}(T) \geq 0$ (resp. $\leq 0$ ) as an operator on $\mathbb{C}^{n} \otimes \mathcal{H}$ if and only if $\mathcal{R}_{\mathrm{R}}(T) \geq 0$ (resp. $\leq 0)$ as an operator on $\mathfrak{S}_{2 n} \otimes \mathcal{H}$.

The proof of both statements is based on the next simple characterization of positive operators on $\mathbb{C}^{n} \otimes \mathcal{H}$.

2.7. Lemma. Let $\mathcal{C}=\left(C_{i j}\right)_{i, j=1}^{n}$ be an operator on $\mathbb{C}^{n} \otimes \mathcal{H}$. The following two conditions are equivalent:

(i) $\mathcal{C} \geq 0$;

(ii) there exists an $n$-tuple $C=\left(C_{1}, C_{2}, \ldots, C_{n}\right)$ of operators on $\mathcal{H}$ such that

$$
C_{i j}=C_{i}^{*} C_{j}, \quad 1 \leq i, j \leq n .
$$

Proof. Since obviously (ii) implies (i), it suffices to show that (i) implies (ii) as well. To this end we first choose a unitary operator $U: \mathbb{C}^{n} \otimes \mathcal{H} \rightarrow \mathcal{H}$. Such unitary operators exist because $\mathcal{H}$ is infinite-dimensional. Next we define $C_{i}: \mathcal{H} \rightarrow \mathcal{H}$ for every $1 \leq i \leq n$ by $C_{i} \xi=U\left(\mathcal{C}^{1 / 2}\left(\sigma_{i} \otimes \xi\right)\right), \xi \in \mathcal{H}$, and observe that

$$
\begin{aligned}
\left\langle C_{i}^{*} C_{j} \xi, \xi\right\rangle & =\left\langle C_{j} \xi, C_{i} \xi\right\rangle_{\mathcal{H}}=\left\langle U\left(\mathcal{C}^{1 / 2}\left(\sigma_{j} \otimes \xi\right)\right), U\left(\mathcal{C}^{1 / 2}\left(\sigma_{i} \otimes \xi\right)\right)\right\rangle_{\mathcal{H}} \\
& =\left\langle\mathcal{C}^{1 / 2}\left(\sigma_{j} \otimes \xi\right), \mathcal{C}^{1 / 2}\left(\sigma_{i} \otimes \xi\right)\right\rangle_{\mathbb{C}^{n} \otimes \mathcal{H}}=\left\langle\mathcal{C}\left(\sigma_{j} \otimes \xi\right), \sigma_{i} \otimes \xi\right\rangle_{\mathbb{C}^{n} \otimes \mathcal{H}} \\
& =\left\langle C_{i j} \xi, \xi\right\rangle_{\mathcal{H}}
\end{aligned}
$$

for any $1 \leq i, j \leq n$ and all $\xi \in \mathcal{H}$. The proof is complete.

Let us now go back to Proposition 2.6 and assume, for instance, that $\varepsilon \mathcal{C}_{\mathrm{R}}(T) \geq 0$, where $\varepsilon$ equals 1 or -1 . Applying Lemma 2.7 to $\mathcal{C}=\varepsilon \mathcal{C}_{\mathrm{R}}(T)$ we get an $n$-tuple $S=\left(S_{1}, S_{2}, \ldots, S_{n}\right)$ such that $\varepsilon\left[T_{i}^{*}, T_{j}\right]=S_{i}^{*} S_{j}, 1 \leq i, j \leq n$. Further, we associate to $S$ the operator $\mathcal{D}(S)$ defined as in (2.5) and notice that

$$
\mathcal{R}_{\mathrm{R}}(T)=\varepsilon \mathcal{D}^{*}(S) \mathcal{D}(S),
$$

whence $\varepsilon \mathcal{R}_{\mathrm{R}}(T) \geq 0$. In a similar way, if $\varepsilon \mathcal{C}_{\mathrm{L}}(T) \geq 0$, we can find an $n$-tuple $S=\left(S_{1}, S_{2}, \ldots, S_{n}\right)$ such that

$$
\mathcal{R}_{\mathrm{L}}(T)=\varepsilon \mathcal{D}(S) \mathcal{D}^{*}(S) .
$$

2.8. We should mention that the $n$-tuples $S$ used in (2.9) and (2.10) are not uniquely defined. However, in some specific situations, we can opt for a natural alternative. The next example reconsiders a result proven in 2], which shows that every subnormal tuple fits into the class of left hyponormal tuples. Specifically, we assume that $N=\left(N_{1}, N_{2}, \ldots, N_{n}\right)$ is a commuting normal $n$-tuple of operators on $\mathcal{H}$ and $\mathcal{K} \subseteq \mathcal{H}$ is an invariant subspace of each $N_{i}, 1 \leq i \leq n$. If $P$ denotes the orthogonal projection of $\mathcal{H}$ onto $\mathcal{K}$, we set $T_{i}=P N_{i} P=N_{i} P$ and $S_{i}=P N_{i}(I-P)$, $1 \leq i \leq n$. Since $N_{i} N_{j}^{*}=N_{j}^{*} N_{i}$, direct calculations show that $\left[T_{i}, T_{j}^{*}\right]=-S_{i} S_{j}^{*}$ for all $1 \leq i, j \leq n$, whence $\mathcal{R}_{\mathrm{L}}(T)=-\mathcal{D}(S) \mathcal{D}^{*}(S)$. 
2.9. Proposition 2.6 and the Bochner-Kodaira identities $\left(B K_{L}\right)$ and $\left(B K_{R}\right)$ can be used to formulate a simple test for two-sided seminormality. We notice that

$$
\mathcal{R}_{\mathrm{L}}(T)-\mathcal{R}_{\mathrm{R}}(T)=I \otimes\left(\Delta_{\mathrm{R}}^{\mathrm{c}}(T)-\Delta_{\mathrm{L}}^{\mathrm{c}}(T)\right)=I \otimes \sum_{i=1}^{n}\left[T_{i}, T_{i}^{*}\right]
$$

Therefore, $T$ is two-sided cohyponormal, i.e., left and right cohyponormal, if and only if

$$
0 \leq \mathcal{R}_{\mathrm{L}}(T) \leq I \otimes \sum_{i=1}^{n}\left[T_{i}, T_{i}^{*}\right]
$$

and $T$ is two-sided hyponormal if and only if

$$
0 \leq \mathcal{R}_{\mathrm{R}}(T) \leq I \otimes \sum_{i=1}^{n}\left[T_{i}^{*}, T_{i}\right]
$$

We should mention that the right-hand sides of (2.12) and (2.13) involve the formal traces of the operator matrices $\mathcal{C}_{\mathrm{L}}(T)$ and $\mathcal{C}_{\mathrm{R}}(T)$, respectively. Equation (2.11) has no natural counterpart in terms of $\mathcal{C}_{\mathrm{L}}(T)$ and $\mathcal{C}_{\mathrm{R}}(T)$.

2.10. The remaining part of this section is concerned with a singular integral model of jointly seminormal pairs of self-adjoint tuples of operators that generalizes to higher dimension the one-dimensional model discovered by D. Xia and J. D. Pincus.

We first recall that the Riesz transforms of a complex-valued function $u$ in the Schwartz space $\mathcal{S}\left(\mathbb{R}^{n}, \mathbb{C}\right)$ are defined by the principal value integrals

$$
R_{i} u(x)=\mathrm{p} . \mathrm{v} \cdot \frac{\Gamma((n+1) / 2)}{\pi^{(n+1) / 2}} \int_{\mathbb{R}^{n}} \frac{x_{i}-y_{i}}{|x-y|^{n+1}} u(y) d y, \quad 1 \leq i \leq n, x \in \mathbb{R}^{n}
$$

For the ease of notation, we let $k_{i}$ denote the kernels

$$
k_{i}(x)=\frac{\Gamma((n+1) / 2)}{\pi^{(n+1) / 2}} \cdot \frac{x_{i}}{|x|^{n+1}}, \quad 1 \leq i \leq n, x \in \mathbb{R}_{0}^{n}=\mathbb{R}^{n} \backslash\{0\},
$$

and write $(2.14)$ as $R_{i} u(x)=k_{i} * u(x), 1 \leq i \leq n$.

In addition, we introduce the operators $M_{i}$ on $\mathcal{S}\left(\mathbb{R}^{n}, \mathbb{C}\right)$ defined as

$$
M_{i} u(x)=x_{i} u(x), \quad 1 \leq i \leq n, x=\left(x_{1}, x_{2}, \ldots, x_{n}\right) \in \mathbb{R}^{n} .
$$

A simple calculation that is left to our reader shows that the commutators $\left[M_{i}, R_{j}\right]$, $1 \leq i, j \leq n$, are singular integral operators, namely,

$$
\left[M_{i}, R_{j}\right] u(x)=k_{i j} * u(x), \quad u \in \mathcal{S}\left(\mathbb{R}^{n}, \mathbb{C}\right), x \in \mathbb{R}^{n}
$$

where the kernels $k_{i j}$ are given by

$$
k_{i j}(x)=x_{i} k_{j}(x)=x_{j} k_{i}(x), \quad x \in \mathbb{R}_{0}^{n},
$$

with $k_{i}$ and $k_{j}$ as in (2.25). In particular, we have $\left[M_{i}, R_{j}\right]=\left[M_{j}, R_{i}\right], 1 \leq i, j \leq n$.

We next form the commutator $n \times n$ matrix $\mathcal{C}=\left(\left[M_{i}, R_{j}\right]\right)_{i, j=1}^{n}$, and consider the corresponding operator on the Schwartz space $\mathcal{S}\left(\mathbb{R}^{n}, \mathbb{C}^{n}\right)$ of $\mathbb{C}^{n}$-valued functions on $\mathbb{R}^{n}$. Actually, using the notation introduced in Section 2.4 and the conventions made there, we may represent every $u \in \mathcal{S}\left(\mathbb{R}^{n}, \mathbb{C}^{n}\right)$ as

$$
u=\sigma_{1} \otimes u_{1}+\sigma_{2} \otimes u_{2}+\cdots+\sigma_{n} \otimes u_{n} \in \mathfrak{S}_{2 n}^{1} \otimes \mathcal{S}\left(\mathbb{R}^{n}, \mathbb{C}\right)
$$


and interpret $\mathcal{C}$ as a linear operator on $\mathfrak{S}_{2 n}^{1} \otimes \mathcal{S}\left(\mathbb{R}^{n}, \mathbb{C}\right)$, namely,

$$
\mathcal{C}=\sum_{i, j=1}^{n} \varepsilon_{i} \varepsilon_{j}^{*} \otimes\left[M_{i}, R_{j}\right]
$$

Finally, on $\mathcal{S}\left(\mathbb{R}^{n}, \mathbb{C}\right)$ we take the inner product inherited from the standard Lebesgue space $L^{2}\left(\mathbb{R}^{n}\right)$ of square integrable complex-valued functions on $\mathbb{R}^{n}$, and then convert $\mathfrak{S}_{2 n}^{1} \otimes \mathcal{S}\left(\mathbb{R}^{n}, \mathbb{C}\right)$ into an inner product space in the usual way. We are now in a position to formulate the main result of this section.

2.11. Theorem. The operator $\mathcal{C}$ defined by (2.20) is positive semidefinite, that is,

$$
\langle\mathcal{C} u, u\rangle \geq 0
$$

for any $u \in \mathcal{S}\left(\mathbb{R}^{n}, \mathbb{C}^{n}\right) \equiv \mathfrak{S}_{2 n}^{1} \otimes \mathcal{S}\left(\mathbb{R}^{n}, \mathbb{C}\right)$.

The proof of Theorem 2.11 given below is based on a non-trivial Fourier transform argument summarized in the next lemma. For a complete proof we refer to Chapter 2, Section 9, in 25]. The form of the Fourier transform used throughout this section is

$$
\hat{u}(\xi)=\int_{\mathbb{R}^{n}} e^{-\sqrt{-1}\langle x, \xi\rangle} u(x) d x, \quad \xi \in \mathbb{R}^{n} .
$$

2.12. Lemma. If $\phi$ is a harmonic polynomial on $\mathbb{R}^{n}$ homogeneous of degree $m \geq 0$, then

$$
\left(\phi(x)|x|^{-\lambda-m}\right)^{\widehat{A}}=\sqrt{-1}^{-m} \pi^{m / 2} 2^{n-\lambda} \frac{\Gamma((m+n-\lambda) / 2)}{\Gamma((m+\lambda) / 2)} \phi(\xi)|\xi|^{-m-n+\lambda},
$$

for any complex number $\lambda \neq n+m+2 k, k=0,1, \ldots$

In particular, we observe that by using (2.22) for the kernels (2.15) we get

$$
\left(R_{i} u\right)^{\widehat{\Upsilon}}(\xi)=-\sqrt{-1} \xi_{i}|\xi|^{-1} \hat{u}(\xi), \quad u \in \mathcal{S}\left(\mathbb{R}^{n}, \mathbb{C}\right), \xi \in \mathbb{R}^{n},
$$

for each $1 \leq i \leq n$, a set of equations that immediately leads to several basic properties of the Riesz transforms. For instance, one gets that each $R_{i}, 1 \leq i \leq n$, extends to a skew-adjoint operator on $L^{2}\left(\mathbb{R}^{n}\right)$, such that $\sum_{i=1}^{n} R_{i}^{*} R_{i}$ equals the identity on $L^{2}\left(\mathbb{R}^{n}\right)$ and $\sum_{i=1}^{n}\left\|R_{i} u\right\|^{2}=\|u\|^{2}$ for any $u \in L^{2}\left(\mathbb{R}^{n}\right)$. More properties of Riesz transforms are discussed in Stein's monographs 33] 34.

As far as we are concerned, we need to compute the Fourier transforms of the kernels $k_{i j}$ defined by (2.18). We assume that $1 \leq i, j \leq n$ are fixed and write

$$
x_{i} x_{j}=\phi_{2}(x)+\phi_{0}(x)|x|^{2},
$$

where

$$
\phi_{2}(x)=x_{i} x_{j}-\frac{\delta_{i j}}{n}|x|^{2} \quad \text { and } \quad \phi_{0}(x)=\frac{\delta_{i j}}{n}
$$

are harmonic polynomials homogeneous of degree 2 and 0 . Using (2.23) in conjunction with (2.22) for $\phi_{2}$ and $\phi_{0}$ with $\lambda=n-1$ we get

$$
\left(x_{i} x_{j}|x|^{-(n+1)}\right)^{\widehat{ }}=-\pi^{n / 2} 2 \frac{\Gamma(3 / 2)}{\Gamma((n+1) / 2)} \phi_{2}(\xi)|\xi|^{-3}+\pi^{n / 2} 2 \frac{\Gamma(1 / 2)}{\Gamma((n-1) / 2)} \phi_{0}(\xi)|\xi|^{-1} \text {. }
$$


Since $\Gamma(1 / 2)=\pi^{1 / 2}, \Gamma(3 / 2)=\pi^{1 / 2} / 2$, and $\Gamma((n-1) / 2)=(2 /(n-1)) \Gamma((n+1) / 2)$, by substituting (2.24) into the previous equation we obtain

$$
\left(x_{i} x_{j}|x|^{-(n+1)}\right)^{\wedge}=\frac{\pi^{(n+1) / 2}}{\Gamma((n+1) / 2)}\left(\delta_{i j}|\xi|^{-1}-\xi_{i} \xi_{j}|\xi|^{-3}\right), \quad \xi \in \mathbb{R}_{0}^{n} .
$$

Combining the last equation with (2.18) and (2.17) we conclude that

$$
\hat{k}_{i j}(\xi)=\delta_{i j}|\xi|^{-1}-\xi_{i} \xi_{j}|\xi|^{-3}, \quad 1 \leq i, j \leq n, \xi \in \mathbb{R}_{0}^{n} .
$$

We are now ready to present the proof of Theorem 2.11. Suppose $u \in \mathcal{S}\left(\mathbb{R}^{n}, \mathbb{C}^{n}\right)$ is given by (2.19) and $\mathcal{C}$ is as in (2.20). Then, by employing some well-known properties of the Fourier transform we have

$$
\begin{aligned}
\langle\mathcal{C} u, u\rangle & =\sum_{i, j=1}^{n}\left\langle k_{i j} * u_{j}, u_{i}\right\rangle_{L^{2}\left(\mathbb{R}^{n}\right)}=(2 \pi)^{-n} \sum_{i, j=1}^{n}\left\langle\hat{k}_{i j} \hat{u}_{j}, \hat{u}_{i}\right\rangle_{L^{2}\left(\mathbb{R}^{n}\right)} \\
& =(2 \pi)^{-n} \int_{\mathbb{R}^{n}} \sum_{i, j=1}^{n} \hat{k}_{i j}(\xi) \hat{u}_{j}(\xi) \overline{\hat{u}}_{i}(\xi) d \xi .
\end{aligned}
$$

Therefore, to prove (2.21) it suffices to check that the integrand in the previous equation is a non-negative function almost everywhere on $\mathbb{R}^{n}$. To this end, we assume that $\xi \in \mathbb{R}_{0}^{n}$ is fixed, set $\hat{u}_{i}(\xi)=\lambda_{i}, 1 \leq i \leq n, \lambda=\left(\lambda_{1}, \lambda_{2}, \ldots, \lambda_{n}\right) \in \mathbb{C}^{n}$, and observe that (2.25) leads to

$$
\begin{aligned}
\sum_{i, j=1}^{n} \hat{k}_{i j}(\xi) \hat{u}_{j}(\xi) \overline{\hat{u}}_{i}(\xi) & =\sum_{i, j=1}^{n}\left(|\xi|^{-1} \delta_{i j} \lambda_{j} \bar{\lambda}_{i}-|\xi|^{-3} \xi_{i} \xi_{j} \lambda_{j} \bar{\lambda}_{i}\right) \\
& =|\xi|^{-1}|\lambda|^{2}-|\xi|^{-3}\left|\langle\xi, \lambda\rangle_{\mathbb{C}^{n}}\right|^{2} \\
& =|\xi|^{-3}\left(|\xi|^{2}|\lambda|^{2}-\left|\langle\xi, \lambda\rangle_{\mathbb{C}^{n}}\right|^{2}\right) .
\end{aligned}
$$

The classical Cauchy inequality clearly concludes the proof.

2.13. Theorem 2.11 will prove essential in developing the model mentioned at the beginning of Section 2.10 .

As a Hilbert space $\mathcal{H}$ we take the Lebesgue space $L^{2}(\Omega)$ where $\Omega$ is a compact subset of $\mathbb{R}^{n}$. We are going to identify $L^{2}(\Omega)$ with the subspace of $L^{2}\left(\mathbb{R}^{n}\right)$ consisting of functions equal to zero on $\mathbb{R}^{n} \backslash \Omega$, and let $P_{\Omega}$ be the orthogonal projection of $L^{2}\left(\mathbb{R}^{n}\right)$ onto $L^{2}(\Omega)$.

Next assume that $a_{1}, a_{2}, \ldots, a_{n}, b \in L^{\infty}(\Omega)$ are given, with $a_{1}, a_{2}, \ldots, a_{n}$ realvalued and $b$ different from zero almost everywhere, and let $A_{i} \in \mathcal{L}\left(L^{2}(\Omega)\right)$ and $B \in \mathcal{L}\left(L^{2}(\Omega)\right)$ denote the operators of pointwise multiplication by $a_{i}, 1 \leq i \leq n$, and $b$, respectively. We will refer to the functions $a_{1}, a_{2}, \ldots, a_{n}$ as secondary parameters, and to the function $b$ as the primary parameter.

We now introduce the self-adjoint $n$-tuples $X=\left(X_{1}, X_{2}, \ldots, X_{n}\right)$ and $Y=$ $\left(Y_{1}, Y_{2}, \ldots, Y_{n}\right)$ of operators on $L^{2}(\Omega)$ by setting $X_{i}=M_{i} \mid L^{2}(\Omega), 1 \leq i \leq n$, with $M_{i}$ given by $(2.16)$, and $Y_{i}=A_{i}-\sqrt{-1} B P_{\Omega} R_{i} P_{\Omega} B^{*}, 1 \leq i \leq n$, where $R_{i}$ are the Riesz operators defined by (2.14) and considered as operators on $L^{2}\left(\mathbb{R}^{n}\right)$.

We claim that the so-defined pair $(X, Y)$ is jointly hyponormal, that is, the corresponding $n \times n$ commutator matrix $\mathcal{C}(X, Y)$ given by $(2.2)$ is positive semidefinite. To prove our claim we first observe that

$$
\left[X_{i}, Y_{j}\right]=\left[X_{j}, Y_{i}\right]=-\sqrt{-1} B P_{\Omega}\left[M_{i}, R_{j}\right] P_{\Omega} B^{*}, \quad 1 \leq i, j \leq n .
$$


Substituting these equations into (2.2) we get

$$
\mathcal{C}(X, Y)=2\left(B P_{\Omega}\left[M_{i}, R_{j}\right] P_{\Omega} B^{*}\right)_{i, j=1}^{n} .
$$

It remains to observe that whenever $u \in \mathfrak{S}_{2 n}^{1} \otimes L^{2}(\Omega) \subset \mathfrak{S}_{2 n}^{1} \otimes L^{2}\left(\mathbb{R}^{n}\right)$ we get $\langle\mathcal{C}(X, Y) u, u\rangle=2\left\langle\mathcal{C}_{\Omega} B^{*} u, B^{*} u\right\rangle$, where $\mathcal{C}_{\Omega}$ is the compression of the operator $\mathcal{C}$ defined by $(2.20)$ to $\mathfrak{S}_{2 n}^{1} \otimes L^{2}(\Omega)$. Since $u$ can be approximated by restrictions to $\Omega$ of functions from $\mathfrak{S}_{2 n}^{1} \otimes \mathcal{S}\left(\mathbb{R}^{n}, \mathbb{C}^{n}\right)$, Theorem 2.11 shows that $\mathcal{C}_{\Omega} \geq 0$, hence $\mathcal{C}(X, Y) \geq 0$.

The reader should be aware that in the case when $n=1$, the previous model coincides with the singular integral model of pure hyponormal operators with onedimensional self-commutators. If $n=1$, there is just one Riesz operator, usually referred to as the Hilbert transform. For details, we refer to [6], [36], and [18].

\section{Putnam type inequalities}

In this section we establish two Putnam type inequalities in higher dimension. We are going to use freely the notations and terminology introduced in Section 2.

3.1. The classical Putnam's inequality, first proved in [30, amounts to

$$
\left\|T^{*} T-T T^{*}\right\| \leq \frac{1}{\pi} \operatorname{area}(\operatorname{spec} T),
$$

where $T$ is a seminormal operator on a Hilbert space $\mathcal{H}$, spec $T \subset \mathbb{C} \equiv \mathbb{R}^{2}$ denotes its spectrum, and area is the two-dimensional Lebesgue measure on $\mathbb{R}^{2}$.

For a seminormal $n$-tuple $T$ of operators on $\mathcal{H}$ one might expect a result of the form

$$
\|\mathcal{C}(T)\| \leq \alpha_{n} \operatorname{vol}_{2 n}(\operatorname{spec} T)^{1 / n},
$$

where $\mathcal{C}(T)$ stands for the self-commutator $\mathcal{C}_{\mathrm{L}}(T)$ or $\mathcal{C}_{\mathrm{R}}(T)$ defined in Section 2.1, $\operatorname{spec} T \subset \mathbb{C}^{n} \equiv \mathbb{R}^{2 n}$ is an appropriate substitute for the spectrum, $\operatorname{vol}_{2 n}$ equals the Lebesgue measure in $\mathbb{R}^{2 n}$, and $\alpha_{n}$ is a universal constant independent of $T$. For commuting tuples, a natural choice for $\operatorname{spec}(T)$ would be the Taylor spectrum. Unfortunately, even for such tuples, the estimate (3.2) is groundless. If $T_{1}$ is a non-normal seminormal operator, for instance, the unilateral shift, and $T_{2}=\cdots=$ $T_{n}=0$ with $n \geq 2$, then obviously (3.2) fails. Therefore, we must either look for a weaker form or impose certain restrictions on $T$. Specifically, let us assume for a while that $T$ is a commuting $n$-tuple and $\operatorname{spec} T$ equals the Taylor spectrum. Since $\operatorname{spec}(T) \subseteq \operatorname{spec} T_{1} \times \operatorname{spec} T_{2} \times \cdots \times \operatorname{spec} T_{n}$, one gets that $\operatorname{vol}_{2 n}(\operatorname{spec} T)^{1 / n}$ is dominated by the geometric mean, and hence by the arithmetic mean of the numbers area $\left(\operatorname{spec} T_{i}\right), 1 \leq i \leq n$. Consequently, a weaker form of (3.2) would be

$$
\|\mathcal{C}(T)\| \leq \beta_{n} \sum_{i=1}^{n} \operatorname{area}\left(\operatorname{spec} T_{i}\right)
$$

There are good reasons to believe that (3.3) is true in general for not necessarily commuting tuples. For instance, whenever $T$ is a two-sided seminormal tuple, by (2.12) or (2.13) in Section 2.9 we get

$$
\|\mathcal{R}(T)\| \leq \sum_{i=1}^{n}\left\|\left[T_{i}, T_{i}^{*}\right]\right\|,
$$


where $\mathcal{R}(T)$ stands for $\mathcal{R}_{\mathrm{L}}(T)$ or $\mathcal{R}_{\mathrm{R}}(T)$. Combining this inequality with (3.1) we easily derive (3.3) with $\beta_{n}=1 / \pi$. As a matter of fact, (3.3) with $\beta_{n}=1 / \pi$ holds under less restrictive conditions.

3.2. Theorem. If $T=\left(T_{1}, T_{2}, \ldots, T_{n}\right)$ is left (resp. right) seminormal, then

$$
\|\mathcal{C}(T)\| \leq \sum_{i=1}^{n}\left\|\left[T_{i}, T_{i}^{*}\right]\right\|
$$

where $\mathcal{C}(T)=\mathcal{C}_{\mathrm{L}}(T)\left(\right.$ resp. $\left.\mathcal{C}(T)=\mathcal{C}_{\mathrm{R}}(T)\right)$.

Proof. We will only prove (3.4) for left seminormal tuples; the proof of the second alternative is similar. As pointed out in Section 2.7, the remainder $\mathcal{R}_{\mathrm{L}}(T)$ associated with a left seminormal tuple $T$ can be decomposed as in (2.10) where $S=\left(S_{1}, S_{2}, \ldots, S_{n}\right)$ is an $n$-tuple of operators on $\mathcal{H}$ such that $\left[T_{i}, T_{j}^{*}\right]=S_{i} S_{j}^{*}$, $1 \leq i, j \leq n$. Since $\mathcal{C}_{\mathrm{L}}(T)$ can be identified with $\mathcal{R}_{\mathrm{L}}(T) \mid \mathfrak{S}_{2 n}^{1} \otimes \mathcal{H}$, we can write $\mathcal{C}_{\mathrm{L}}(T)$ as $\mathcal{C}_{\mathrm{L}}(T)=\mathcal{D}^{(0)}(S) \mathcal{D}^{(0) *}(S)$, where $\mathcal{D}^{(0)}(S)=\mathcal{D}(S) \mid \mathfrak{S}_{2 n}^{0} \otimes \mathcal{H}: \mathfrak{S}_{2 n}^{0} \otimes \mathcal{H} \rightarrow$ $\mathfrak{S}_{2 n}^{1} \otimes \mathcal{H}$. Therefore,

$$
\left\|\mathcal{C}_{\mathrm{L}}(T)\right\|=\left\|\mathcal{D}^{(0)}(S)\right\|^{2} .
$$

On the other hand, if $\xi \in \mathcal{H} \cong \mathfrak{S}_{2 n}^{0} \times \mathcal{H}$, then

$$
\left\|\mathcal{D}^{(0)}(S) \xi\right\|_{\mathfrak{S}_{2 n}^{1} \otimes \mathcal{H}}^{2}=\left\|\sum_{i=1}^{n} \sigma_{i} \otimes S_{i} \xi\right\|_{\mathfrak{S}_{2 n}^{1} \otimes \mathcal{H}}^{2}=\sum_{i=1}^{n}\left\|S_{i} \xi\right\|_{\mathcal{H}}^{2},
$$

hence

$$
\left\|\mathcal{D}^{(0)}(S)\right\|^{2} \leq \sum_{i=1}^{n}\left\|S_{i}\right\|^{2}=\sum_{i=1}^{n}\left\|S_{i} S_{i}^{*}\right\|=\sum_{i=1}^{n}\left\|\left[T_{i}, T_{i}^{*}\right]\right\| .
$$

Inequality (3.4) follows from (3.5) and (3.6).

3.3. Our next goal is to set up a self-commutator inequality for the Riesz transform model discussed in Section 2.13. If $n=1$, that model produces the real and imaginary parts $X$ and $Y$ of a hyponormal operator that converts (3.1) into an equality. Actually we have

$$
\|\mathcal{C}(X, Y)\|=\frac{2}{\pi}\|b\|_{L^{2}(\Omega)}^{2},
$$

where $b \in L^{2}(\Omega)$ is the primary parameter. Since in higher dimension we do not expect a result as definite as (3.7), we limit ourselves to generalizing a consequence of (3.7), namely,

$$
\|\mathcal{C}(X, Y)\| \leq \frac{2}{\pi}\|b\|_{L^{\infty}(\Omega)}^{2} \text { length }(\Omega),
$$

where length stands for the Lebesgue measure on $\mathbb{R}$.

Suppose now that $n \geq 1$, and let $\Omega \subset \mathbb{R}^{n}, X=\left(X_{1}, X_{2}, \ldots, X_{n}\right)$ and $Y=$ $\left(Y_{1}, Y_{2}, \ldots, Y_{n}\right)$ be as in Section 2.13. We associate to $\Omega$ the quantity

$$
\Lambda(\Omega)=\sup _{x \in \Omega} \int_{\Omega}|x-y|^{-(n-1)} d y .
$$

As a generalization of (3.8) we have the following result. 
3.4. Theorem. The Riesz transform model satisfies the inequality

$$
\|\mathcal{C}(X, Y)\| \leq 2 \frac{\Gamma((n+1) / 2)}{\pi^{(n+1) / 2}}\|b\|_{L^{\infty}(\Omega)}^{2} \Lambda(\Omega)
$$

where $b \in L^{\infty}(\Omega)$ is the primary parameter.

Before proceeding with a proof of Theorem 3.4 we want to mention an inequality proved in 20] that can be used to estimate the constant $\Lambda(\Omega)$. To formulate it, we suppose that $k: \mathbb{R}_{0}^{n} \rightarrow \mathbb{R}$ is a continuous function satisfying the homogeneity condition $k(t x)=t^{-\kappa n} k(x), t \in(0, \infty), x \in \mathbb{R}_{0}^{n}$, where $0<\kappa<1$, and let $A(k)$ be defined by

$$
A(k)=\frac{1}{1-\kappa}\left[\operatorname{vol}_{n}\left\{x \in \mathbb{R}_{0}^{n}:|k(x)| \geq 1\right\}\right]^{\kappa} .
$$

3.5. Lemma ([20, Proposition 1.7]). If $\chi \in L^{1}\left(\mathbb{R}^{n}\right) \cap L^{\infty}\left(\mathbb{R}^{n}\right)$, then

$$
\|k * \chi\|_{L^{\infty}\left(\mathbb{R}^{n}\right)} \leq A(k)\|\chi\|_{L^{1}\left(\mathbb{R}^{n}\right)}^{1-\kappa}\|\chi\|_{L^{\infty}\left(\mathbb{R}^{n}\right)}^{\kappa} .
$$

In particular, by taking the kernel $k(x)=|x|^{-(n-1)}, x \in \mathbb{R}_{0}^{n}$, and setting $\chi$ equal to the characteristic function of the set $\Omega$, from Lemma 3.5 we get

$$
\Lambda(\Omega) \leq n\left(\operatorname{vol}_{n} \mathbb{B}^{n}\right)^{(n-1) / n}\left(\operatorname{vol}_{n} \Omega\right)^{1 / n}
$$

where $\mathbb{B}^{n}$ is the closed unit ball in $\mathbb{R}^{n}$.

3.6. We are now ready to prove Theorem 3.4. Assume that $u=\sum_{i=1}^{n} \sigma_{i} \otimes u_{i} \in$ $\mathbb{C}^{n} \otimes L^{2}(\Omega)$ is fixed. By $(2.26)$ and $(2.17)$ we get

$$
\langle\mathcal{C}(X, Y) u, u\rangle=2 \sum_{i, j=1}^{n}\left\langle K_{i j}\left(\bar{b} u_{j}\right), \bar{b} u_{i}\right\rangle_{L^{2}(\Omega)}
$$

where $K_{i j}: L^{2}(\Omega) \rightarrow L^{2}(\Omega)$ are the convolution operators defined by

$$
K_{i j} u(x)=\int_{\Omega} k_{i j}(x-y) u(y) d y, \quad x \in \Omega, u \in L^{2}(\Omega),
$$

with the kernels $k_{i j}$ given by (2.18), that is,

$$
k_{i j}(x)=\frac{\Gamma((n+1) / 2)}{\pi^{(n+1) / 2}} \cdot \frac{x_{i} x_{j}}{|x|^{n+1}}, \quad x \in \mathbb{R}_{0}^{n} .
$$

The right-hand side of (3.12) can now be expressed as a double integral,

$$
\langle\mathcal{C}(X, Y) u, u\rangle=2 \frac{\Gamma((n+1) / 2)}{\pi^{(n+1) / 2}} \int_{\Omega} \int_{\Omega} \frac{\langle x-y, b(y) \bar{u}(y)\rangle_{\mathbb{C}^{n}}\langle x-y, \bar{b}(x) u(x)\rangle_{\mathbb{C}^{n}}}{|x-y|^{n+1}} d y d x
$$

where $\langle x-y, b(y) \bar{u}(y)\rangle_{\mathbb{C}^{n}}=\sum_{j=1}^{n}\left(x_{j}-y_{j}\right) \bar{b}(y) u_{j}(y)$ and $\langle x-y, \bar{b}(x) u(x)\rangle_{\mathbb{C}^{n}}=$ $\sum_{i=1}^{n}\left(x_{i}-y_{i}\right) \bar{b}(x) u_{i}(x)$. Next using the Cauchy inequality for these inner products in $\mathbb{C}^{n}$ we have

$$
\left|\langle x-y, b(y) \bar{u}(y)\rangle_{\mathbb{C}^{n}}\right| \leq|x-y||b(y)|\|u(y)\|_{\mathbb{C}^{n}}
$$

and

$$
\langle x-y, \bar{b}(x) u(x)\rangle_{\mathbb{C}^{n}} \leq|x-y||b(x)|\|u(x)\|_{\mathbb{C}^{n}}
$$


Based on Section 2.13 we know that $\langle\mathcal{C}(X, Y) u, u\rangle \geq 0$. From (3.15), (3.16), and (3.17) we get the estimate

$$
\langle\mathcal{C}(X, Y) u, u\rangle \leq 2 \frac{\Gamma((n+1) / 2)}{\pi^{(n+1) / 2}} \int_{\Omega} \int_{\Omega} \frac{|b(x)||b(y)|\|u(x)\|_{\mathbb{C}^{n}}\|u(y)\|_{\mathbb{C}^{n}}}{|x-y|^{n-1}} d y d x .
$$

The integrand in the right-hand side of (3.18) is a product of two functions $v$ and $w$ in $L^{2}(\Omega \times \Omega)$, namely, $v(x, y)=|b(x)|\|u(x)\|_{\mathbb{C}^{n}} /|x-y|^{(n-1) / 2}$ and $w(x, y)=$ $|b(y)|\|u(y)\|_{\mathbb{C}^{n}} /|x-y|^{(n-1) / 2}=v(y, x)$. Their norms in $L^{2}(\Omega \times \Omega)$ are obviously equal. More precisely we have

$$
\|v\|_{L^{2}(\Omega \times \Omega)}^{2}=\int_{\Omega}|b(x)|^{2}\|u(x)\|_{\mathbb{C}^{n}}^{2}\left(\int_{\Omega}|x-y|^{-(n-1)} d y\right) d x .
$$

Now using the Cauchy inequality in $L^{2}(\Omega \times \Omega)$, from (3.18) and (3.19) we get

$$
\langle\mathcal{C}(X, Y) u, u\rangle \leq 2 \frac{\Gamma((n+1) / 2)}{\pi^{(n+1) / 2}} \int_{\Omega}|b(x)|^{2}\|u(x)\|_{\mathbb{C}^{n}}^{2}\left(\int_{\Omega}|x-y|^{-(n-1)} d y\right) d x .
$$

Further, we combine (3.20) with the obvious estimates $|b(x)| \leq\|b\|_{L^{\infty}(\Omega)}$ almost everywhere on $\Omega$, and $\int_{\Omega}|x-y|^{-(n-1)} d y \leq \Lambda(\Omega)$ for all $x \in \Omega$, and get

$$
\langle\mathcal{C}(X, Y) u, u\rangle \leq 2 \frac{\Gamma((n+1) / 2)}{\pi^{(n+1) / 2}} \Lambda(\Omega)\|b\|_{L^{\infty}(\Omega)}^{2}\|u\|_{\mathbb{C}^{n} \otimes L^{2}(\Omega)}^{2} .
$$

The last inequality concludes the proof of Theorem 3.4.

As a final comment, we want to mention that by combining the estimates (3.10) and (3.11) we get an inequality that resembles the inequality established in [7] for doubly-commuting hyponormal tuples. However, the Riesz transform model does not produce a doubly-commuting tuple, so Theorem 3.6 somehow complements the investigation made in [7].

\section{REFERENCES}

[1] H. Alexander, Projections of polynomial hulls, J. Funct. Anal. 13 (1973), 13-19. MR 49:3209

[2] A. Athavale, On joint hyponormal operators, Proc. Amer. Math. Soc. 103 (1988), 417-423. MR 89f: 47033

[3] S. Axler, and J. H. Shapiro, Putnam's theorem, Alexander's spectral area estimate, and VMO, Math. Ann. 271 (1985), 161-183. MR 87b:30053

[4] N. Berline, E. Getzler, and M. Vergne, Heat kernels and Dirac operators, in Grundlehren der mathematischen Wissenschaften, 298, Springer-Verlag, Berlin, Heidelberg, New York, 1992. MR 94e:58130

[5] F. Brackx, R. Delanghe, and F. Sommen, Clifford Analysis, Pitman Research Notes in Mathematics Series, 76, 1982. MR 85j:30103

[6] K. F. Clancey, Seminormal Operators, Lecture Notes in Math., 742, Springer-Verlag, 1979. MR 81c: 47002

[7] M. Cho, R. E. Curto, T. Huruya, and W. Zelazko, Cartesian form of Putnam's inequality for doubly commuting n-tuples, Indiana Univ. Math. J. 49 (2000), 1437-1448.

[8] J. B. Conway, Subnormal Operators, Pitman, Boston, 1981. MR 83i:47030

[9] R. E. Curto, Joint hyponormality: a bridge between hyponormality and subnormality, in Operator Theory, Operator Algebras and Applications (W. B. Arveson and R. G. Douglas, eds.); part 2, Proc. Sympos. Pure Math. 51 (1990), 69-91. MR 91k:47049

[10] R. E. Curto, and R. Jian, A matricial identity involving the self-commutator of a commuting n-tuple, Proc. Amer. Math. Soc. 121 (1994), 461-464. MR 94h:47063

[11] R. E. Curto, P. A. Muhly, and J. Xia, Hyponormal pairs of commuting operators, in Operator Theory: Advances and Applications, 35, Birkhäuser Verlag, 1988, 1-22. MR 90m:47037 
[12] R. G. Douglas, V. Paulsen, and K. Yan, Operator theory and algebraic geometry, Bull. Amer. Math. Soc. 20 (1988), 67-71.

[13] J. E. Gilbert, and M. A. M. Murray, Clifford Algebras and Dirac Operators in Harmonic Analysis, Cambridge Studies in Advanced Mathematics, 26, Cambridge University Press, 1991. MR 93e: 42027

[14] T. Kato, Smooth operators and commutators, Studia Math. 31 (1968), 531-546. MR 38:2631

[15] H. B. Lawson and M.-L. Michelsohn, Spin Geometry, Princeton Mathematical Series, 38, Princeton University Press, Princeton, 1989. MR 91g:53001

[16] M. Martin, Joint seminormality and Dirac operators, Integral Equations Operator Theory 30 (1998), 101-121. MR 99f:58186

[17] M. Martin, Higher-dimensional Ahlfors-Beurling inequalities in Clifford analysis, Proc. Amer. Math. Soc. 126 (1998), 2863-2871. MR 99a:30045

[18] M. Martin and M. Putinar, Lectures on Hyponormal Operators, Operator Theory: Advances and Applications, 39, Birkhäuser Verlag, Basel, 1989. MR 91c:47041]

[19] M. Martin, and N. Salinas, Weitzenböck type formulas and joint seminormality, in Contemporary Math., Amer. Math. Soc. 212 (1998), 157-167. MR 98j:47054

[20] M. Martin, and P. Szeptycki, Sharp inequalities for convolution operators with homogeneous kernels and applications, Indiana Univ. Math. J. 46 (1997), 975-988. MR 99b:42017

[21] S. McCullough and V. Paulsen, A note on joint hyponormality, Proc. Amer. Math. Soc. 107 (1989), 187-195. MR 90a:47062

[22] A. McIntosh and A. Pryde, A functional calculus for several commuting operators, Indiana Univ. Math. J. 36 (1987), 431-439. MR 88i:47007

[23] M. Mitrea, Singular Integrals, Hardy Spaces, and Clifford Wavelets, Lecture Notes in Mathematics, 1575, Springer-Verlag, Heidelberg, 1994. MR 96e:31005

[24] P. S. Muhly, A note on commutators and singular integrals, Proc. Amer. Math. Soc. 54 (1976), 117-121. MR 52:15089

[25] B. E. Petersen, Introduction to the Fourier Transform and Pseudo-Differential Operators, Monographs and Studies in Mathematics, 19, Pitman, Boston, 1983. MR 85d:46001

[26] J. D. Pincus, Commutators and systems of singular integral equations, Acta Math. 121 (1968), 219-249. MR 39:2026

[27] J. D. Pincus, and D. Xia, Mosaic and principal function of hyponormal and semihyponormal operators, Integral Equations Operator Theory 4 (1981), 134-150. MR 84d:47030

[28] J. D. Pincus, D. Xia, and J. Xia, The analytic model of a hyponormal operator with rank one self-commutator, Integral Equations Operator Theory 7 (1984), 516-535. MR 87d:47030a

[29] C. R. Putnam, Commutation Properties of Hilbert Space Operators and Related Topics, Springer-Verlag, Berlin, Heidelberg, New York, 1967. MR 36:707

[30] C. R. Putnam, An inequality for the area of hyponormal spectra, Math. Z. 116 (1970), 323330. MR 42:5085

[31] J. Ryan (editor), Clifford Algebras in Analysis and Related Topics, CRC Press, Boca Raton, 1996. MR 97d:30065

[32] J. Ryan, Dirac operators, conformal transformations and aspects of classical harmonic analysis, Journal of Lie Theory 8 (1998), 67-82. MR 99h:42029

[33] E. M. Stein, Singular Integrals and Differentiability Properties of Functions, Princeton Univ. Press, Princeton, NJ, 1970. MR 44:7280

[34] E. M. Stein, Harmonic Analysis: Real-Variable Methods, Orthogonality, and Oscillatory Integrals, Princeton Univ. Press, Princeton, NJ, 1993. MR 95c:42002

[35] D. Xia, On non-normal operators. I, Chinese J. Math. 3 (1963), 232-246; II, Acta Math. Sinica 21 (1987), 103-108. MR 26:6773; MR 80g:47020

[36] D. Xia, Spectral Theory of Hyponormal Operators, Birkhäuser Verlag, Basel-BostonStuttgart, 1983. MR 87j:47036

[37] D. Xia, On some classes of hyponormal tuples of commuting operators, in Operator Theory: Advances and Applications, 48, Birkhäuser Verlag, 1990, pp. 423-448. MR 94g:47029

Department of Mathematics, Baker University, Baldwin City, Kansas 66006

E-mail address: mmartin@harvey.bakeru.edu 